\title{
Rekurrenz - Evokation - Konnotation: Bausteine für eine strukturalistische Analyse literarischer Texte
}

\author{
Clemens Knobloch
}

Angenommen: 7. Januar 2021 / Online publiziert: 26. Januar 2021

(C) Der/die Autor(en) 2021

Zusammenfassung Über welche theoretischen und methodologischen Ressourcen verfügt ein (strukturalistisch sozialisierter) Linguist für die Analyse dessen, was einen literarischen Text ausmacht? Von zwei Gesichtspunkten her geht der Beitrag dieser Frage nach: Einmal von Eugenio Coserius textlinguistischer Reflexion der Besonderheiten von Literatur, mit dem Schwerpunkt auf geordneter Rekurrenz von Elementen in allen Ebenen, und zum anderen von den Theorien des US-Sprachphilosophen und Literaturkritikers Kenneth Burke. Illustriert werden die Deutungsverfahren an Beispielen aus Lyrik und Prosa.

Schlüsselwörter Literarische Texte · Geordnete Rekurrenz · Konnotation · Deutungsverfahren · Eugenio Coseriu · Kenneth Burke

\section{Recurrence - Evocation - Connotation: Components for a Structuralist Analysis of Literary Texts}

\begin{abstract}
What are the theoretical and methodological resources of a linguist trained in structural methods, for the detection of those traits of text that are specifically literary traits? This question is approached from two angles: First from the angle of Eugenio Coseriu's version of text linguistics (focussing on recurrent returns of units on all levels), and second by using theories and methods developed by the USphilosopher of language and literary critic Kenneth Burke. Some illustrations from poetry and prose are provided.
\end{abstract}

Keywords Literary Text · Connotation · Recurrent Returns · Sign Relations · Eugenio Coseriu · Kenneth Burke

\footnotetext{
Der abgedruckte Text bewahrt den Duktus des Vortrags, auf den er zurückgeht.

C. Knobloch $(\bowtie)$

Germanistisches Seminar, Linguistik, Universität Siegen, Siegen, Deutschland

E-Mail: knobloch@germanistik.uni-siegen.de
} 
Bei der dichterischen Sprache kommt nichts hinzu, was nicht in der Sprache schlechthin bereits vorhanden wäre. Jedoch kommt in der Dichtung vieles von dem voll zur Entfaltung, was in anderen Modalitäten des Sprachgebrauchs sozusagen >ungenutzt bereitsteht $<$. [...] Die Dichtung - und unter Dichtung verstehe ich nicht nur die Poesie im engeren Sinne, sondern die Literatur als Kunst ist der Ort der Entfaltung der funktionellen Vollkommenheit der Sprache. Der dichterische Sprachgebrauch ist nicht etwa eine Abweichung vom >normalen< Sprachgebrauch, genau das Umgekehrte ist der Fall: Alle anderen Modalitäten der Sprache wie z. B. die Alltagssprache oder die Wissenschaftssprache [...] stellen Abweichungen gegenüber der totalen Sprache dar, gegenüber der Sprache schlechthin. Wenn man von Reduktion sprechen darf, so im Fall der verschiedenen Arten des nicht-dichterischen Sprachgebrauchs, denn dort werden viele Sprachfunktionen aufgehoben, >entaktualisiert<, die beim dichterischen Sprechen vollständig präsent sind. Im praktisch ausgerichteten Sprechen gehen wir gewissermaßen >durch das Zeichen hindurch < direkt auf die bezeichnete Sache hin, auf die unsere Aufmerksamkeit gerichtet ist. (Coseriu 1981, S. 110f.)

\section{Vorbemerkung}

Coserius Bekenntnis zur Vollkommenheit der dichterischen Sprache (gegenüber den Alltags- und sonstigen reduzierten Varianten des Sprechens) klingt heute pathetisch und bildungsreligiös, obwohl es genau 40 Jahre alt ist. In den frühen 1980erJahren konnte man sich noch darauf verlassen, dass eine solche Aufwertung literarischer Sprache (sagen wir) ankommt beim relevanten Publikum. Das ist heute durchaus nicht mehr sicher - und verweist auf den Umstand, dass vor allem die evaluativen, bewertenden Bezugssysteme (und just die sind »ultimativ«) einem raschen historischen Wandel unterliegen. Der letzte Horizont aller Texte besteht aus den herrschenden Erfahrungen und Erwartungen des Publikums. Wenn sich deren Balance ernsthaft verschiebt, dann bleibt auch für literarische Texte nichts, wie es ist. Diese historische Dimension literarischer Texte muss ich leider ausblenden. Was ich zu sagen habe, ist daher synchronisch vereinfacht.

Was braucht man, um sich als Linguist sinnvoll mit einem literarischen Text zu beschäftigen? Man braucht zunächst zweierlei. Einmal so etwas wie eine Texttheorie, die sich möglichst weit entfernt von unserer alltäglichen Sprachideologie, von der Art und Weise, wie man sich als Sprecher das Funktionieren »seiner « Sprache zurechtlegt. $\mathrm{Zu}$ dieser alltäglichen Sprachideologie gehören solche Annahmen wie: Texte setzen sich aus (referierenden und prädizierenden) Sprachzeichen mit ihren Systembedeutungen zusammen, und die können wir in einem Wörterbuch nachschlagen. Von dieser Alltagsvorstellung der Sprachverwendung (die, merkwürdig genug, auch von vielen Linguisten geteilt wird) führt kein Weg zur Analyse literarischer Texte.

Das zweite, was wir brauchen, ist eine Methodologie für die Analyse dessen, was die literarischen Qualitäten eines Textes ausmacht. Dazu gleich mehr.

Bei literarischen Texten kommt zu den üblichen und sich durch alle Texte ziehenden Redefunktionen (etwa Ausdruck - Appell - Darstellung in Bühlers berühmtem 
Organonmodell) eine weitere Sprachfunktion hinzu, so heißt es der Tradition der strukturalistischen Literaturanalyse: die poetische oder ästhetische Sprachfunktion. $\mathrm{Zu}$ unserer alltäglichen Sprachideologie gesellt sich eine alltägliche Literaturideologie. In dem sicherlich bekanntesten Text über dieses Thema, in Roman Jakobsons »Linguistik und Poetik«, heißt es:

Die Einstellung auf die BOTSCHAFT als solche, die Ausrichtung auf die Botschaft um ihrer selbst willen, stellt die POETISCHE Funktion der Sprache dar. (Jakobson 1960, zit. nach Jakobson 1979, S. 92)

Der Leitgedanke in Roman Jakobsons klassischem Text ist erkennbar, dass die konzentrierte Arbeit an der Form der Nachricht (das Modell ist durchgehend informationstheoretisch) einen Text zum literarischen macht. Denn andere Texte (Gebrauchstexte, Brief, fachliche Abhandlungen etc.) etablieren zwar auch Normen auf der Formebene, sind aber erkennbar durch diese Form hindurch auf das Gemeinte, auf »die Sache« gerichtet.

Jakobsons Lehre von der poetischen Konzentration auf die Form der Nachricht vernachlässigt einiges. Coseriu (1981, S. 56ff.), auf den ich mich hier weitgehend stütze, argumentiert etwa folgendermaßen:

a) Eine durchdachte und perfektionierte Form haben auch anspruchsvolle Werbetexte, politische Reden, wissenschaftliche Abhandlungen, sie ist nicht spezifisch für ästhetische Texte, sondern für kalkulierte Schriftlichkeit und geplante Mündlichkeit; selbst grammatischen und syntaktischen und sonstigen Parallelismus (für Jakobson zentral) findet man nicht nur im Märchen und in der Lyrik, sondern auch in Kochrezepten.

b) Jakobsons Fokus auf die Form der Nachricht würde implizieren, dass man einen ästhetischen und literarischen Inhalt gewissermaßen auch in einer anderen, nichtliterarischen Textform kodieren könnte. Das sei indessen nicht der Fall, die Besonderheit eines literarischen Textes liege ebenso im Inhalt wie in der Form.

Neben diesem etwas kruden nachrichtentheoretischen Element in Jakobsons Poetik gibt es einen weiteren, etwas komplexeren Baustein, der immer wieder zitiert wird. Er lautet: Die Gemeinsamkeit poetisch-ästhetischer Verfahren der Textualisierung bestehe darin, das Äquivalenzprinzip von der paradigmatischen Ebene der Selektion (wo es immer gilt: in allen Ebenen der Paradigmatik können Zeichen nur durch andere Zeichen ersetzt werden, die in irgendeiner Hinsicht äquivalent sind) auf die syntagmatische Ebene der Kombination zu projizieren. Was damit gemeint ist, werde ich gleich an einem banalen Beispiel erklären. Jedenfalls brauchen wir diesen Baustein aus Jakobsons Theorie und werden ihn in die Analyse-Methodologie einfügen. Er ist weit mehr als eine bloße linguistische Definition des Reims, der bestenfalls die auffälligste Verkörperung für diese Projektion von paradigmatischen Äquivalenzen in die Syntagmatik ist.

Weiterhin braucht es für einen sprachwissenschaftlichen Blick auf literarische Texte auch einen Baustein, der nicht aus der strukturalistischen Tradition stammt und dafür muss ich etwas weiter ausholen. 


\section{Exkurs: Sprache und Literatur als sozialwissenschaftliche »Lebensmittel«}

Gegen Ende der Buddenbrooks lässt Thomas Mann den sensiblen Hanno Buddenbrook an einer gespenstischen Lateinstunde teilnehmen. Fast alle Schüler haben einen Spickzettel, aus dem sie die Übersetzung des Textes, die sie eigentlich selbst vornehmen sollen, ablesen. Und, oh Wunder, im Ansehen bei den Mitschülern und in ihrem Selbstbild gilt es als »Erfolg «, den dümmlichen Lehrer hereingelegt zu haben, und als (beschämender) »Misserfolg «, durch eigenes Bemühen den Erwartungen des Lehrers nicht oder soeben zu genügen. Im Unterschied zu seinen lebenstüchtigeren Mitschülern registriert der junge Hanno diese Seltsamkeit.

Diese Szene schildert Gustav Ichheiser am Ende seiner 1930 erschienenen Kritik des Erfolgs (Ichheiser 1930), das Buch ist so etwas wie ein heimlicher Klassiker der modernen Erfolgssoziologie, und die Buddenbrooks-Szene wird nur dann aussagekräftig, wenn man die dort praktisch gelebten Kriterien für »Erfolg « mit der offiziösen Rationalisierung von »Erfolg« vergleicht. Die nämlich setzt den Erfolg mit der Leistung des Einzelnen gleich, er ist gewissermaßen die Belohnung für besondere Leistungen. Die Realität, so die Pointe bei Thomas Mann wie bei Gustav Ichheiser, sieht anders aus: Als Erfolg gilt auch, was einem per Zufall, Gelegenheit, Erbschaft, Betrug, Raub etc. zugefallen ist, während individuelle Leistungen gewöhnlich wenig zum sozialen Erfolg beitragen (vgl. Neckel 2008).

In Umrissen sichtbar wird hinter dieser Konstellation eine Methodologie der wechselseitigen Aufklärung von Alltagssprache, Literatur und Sozialwissenschaft. Die literarisch ausgebaute Alltagssprache ist ein höchst biegsames Medium und vermag die subtilsten sozialen Verhältnisse freizulegen, und sie kann das weitaus genauer als die abstrakte und reifizierte Fachsprache der Sozialwissenschaften. Ersichtlich taugt nicht nur die strukturalistische Methodik dazu, Eigenschaften literarischer Texte freizulegen. Literarische Texte können ihrerseits auch im Blick darauf gelesen werden, was sie an verborgenen Implikationen alltagssprachlicher Ausdrücke freilegen.

Fritz Heider (1980 [1958]), einer der Pioniere der modernen Sozialpsychologie, empfiehlt zur Aufklärung unserer naiven Psychologie und Alltagstheorie (im Anschluss an Ichheiser) ein Verfahren, das einerseits unsere alltäglichen und praxisnahen sprachlichen Ausdrücke zu explizieren sucht, dass andererseits Anekdoten, Redensarten, wiederkehrende literarische Konstellationen als Teilexplikation verborgener, von den Akteuren selbst nicht explizierbarer Relationen innerhalb unserer Alltagssprache wertet. Die »Erfolg «-Episode aus den Buddenbrooks ist dafür ein Beispiel. Kenneth Burke hat für den Zusammenhang, der Heider vorschwebt, die Formel: »the social orders that are implicit in any given complex verbal structure « (Burke 1966, S. 379). »Erfolg«, so ließe sich resümieren, ist selbst ein hoch reflexiver Ausdruck, der bereits metapragmatisch Bezug nimmt auf sprachliche Praktiken, die er rekodiert und konnotativ bis zur Unkenntlichkeit verändert. Man vergleiche eine praxisnahe und alltägliche Formel wie »A hat keine Lust $\mathrm{zu} \mathrm{X}$ « mit der völlig anders konnotierten metapragmatischen Formel »A leidet unter Anhedonie «.

Die Pointe in diesem Zusammenhang lautet, dass Texte (nicht nur, aber auch: literarische Texte) das Rohmaterial sind, aus dem die Sprachwissenschaft ihre Er- 
kenntnisse zieht. Es ist also durchaus nicht so, dass linguistische Analyseverfahren per se geeignet wären, auf literarische Texte angewandt zu werden. Sie können nur das freilegen, was sie zuvor aus literarischen Texten über deren Besonderheit gelernt haben. So gesehen ist es unglücklich, dass die konventionelle Sprachwissenschaft sich weit mehr am Ideal der logisch vollkommenen Darstellung als am Ideal der literarischen Dramaturgie entwickelt hat, und es ist kein Zufall, dass Sprachtheoretiker den Umgang mit literarischen Texten weitgehend von Literaturkritikern und Literaturtheoretikern zu lernen hatten. Und dass es in unserem sprachlich-sozialen Alltag eher dramaturgisch als logisch zugeht, scheint ebenfalls einleuchtend zu sein. »Terministic screens « nennt Kenneth Burke (1966, S. 44-62) die meist zirkulären konzeptuellen Netzwerke, mittels derer wir unsere Beobachtungen sortieren und unsere Handlungen anleiten. Sie liefern das inferentielle Gerüst für explizit sprachliches »Framing « von Situationen und Handlungen (bei Burke wie auch bei dem von Burke beeinflussten Erving Goffman). Derselbe Traum wird völlig unterschiedlich gefiltert und gefärbt, wenn wir ihn einem Freudianer, einem Jungianer oder einem anderen Psychotherapeuten erzählen (und Burke (1966, S. 46) fügt süffisant hinzu, die meisten Patienten würden schnell lernen, die Art von Träumen zu haben, die zu den terministischen Netzen ihrer Analytiker passen!). Was wir in unserer alltäglichen Wirklichkeit direkt zu beobachten meinen, dass hängt nicht zu geringen Teilen an den Möglichkeiten des Netzes von Beobachtungsbegriffen, dessen wir uns bedienen. Beobachtungen, die wir zu teilen glauben, sind womöglich eher den inferentiellen Netzen unserer Terminologien zuzurechnen als den wahrgenommenen »Rohdaten«. Die Analyse solcher Netze, ihrer implikativen und inferentiellen Verbindungen, ist gleichermaßen aufschlussreich für die Theorie der Alltagssprache wie für unsere »naive Psychologie« (Heider 1980 [1958]). Und erst recht natürlich für unsere literarischen Texte, deren Wirkung auf den Leser ausschließlich auf den textuell implementierten terministischen Netzwerken beruht. Aus diesem Grunde wäre ein literarischer Text, den wir mit Hilfe unserer alltäglichen terministischen Deutungsroutinen reibungsfrei bearbeiten könnten, eher ein schlechter Text. Und das gilt auch für einen literarischen Text, der etwa widerspruchsfrei ein freudianisches (oder anderes) wissenschaftliches Deutungsnetzwerk bedienen bzw. illustrieren würde.

Ein gemeinsamer Nenner der vielen verschiedenen Motive, aus denen man Literatur liest (Unterhaltung, Spannung, Entspannung, Ablenkung etc.), dürfte darin bestehen, dass man mit Hilfe der Lektüre bestimmte Dinge anders sieht als zuvor, Erfahrungen anders deutet oder einschätzt etc., kurz: Unsere terministischen Deutungsroutinen werden verunsichert und in Bewegung versetzt.

Das ist auch der Unterschied zwischen Literatur und den (sagen wir) literarischen Hausmannskost-Formen wie Sprichwörtern, Redensarten, Anekdoten, Situationsformeln etc., die Kenneth Burke in »Literature as equipment for living « (Burke 1973 [1941], S. 293-304) in Stellung bringt und in ihrer Funktion als praktisch bewältigende »Überschriften« für zahlreiche, auch sehr unterschiedliche Situationen mit literarischen Texten vergleicht. Sprichwörter fungieren als Aufforderung, Mahnung, Trost, Rache, Schadenfreude, Prognose etc., und auch literarische Textformate können solchermaßen dramaturgisch auf typisierte soziale Situationen bezogen sein. Ebenso wie Predigten, politische Reden und andere Formate zur Bewältigung ty- 
pisierter Situationen. Es ist natürlich eine recht buntscheckige Textsammlung, die solchermaßen zusammengestellt werden kann, und Burke spottet:

The new alignment will outrage in particular those persons who take the division of faculties in our universities to be an exact replica of the way in which God himself divided up the universe. (Burke 1973 [1941], S. 303)

Was für gute Literatur gilt, so ließe sich Burkes Position resümieren, sollte auch für gute Literaturanalysen gelten: Sie sollten dazu beitragen, dass sich eingefahrene und eingelebte Deutungsroutinen »entautomatisieren « lassen (vgl. Abschnitt 6). »Perspective by incongruity «, so der Schlachtruf, nur durch neue, unpassende terministische Kombinationen erkennen wir die eigene Abhängigkeit von altäglichen Deutungsroutinen (und auch die Wissenschaften haben solche Routinen!) und bekommen eine Chance, die Dinge versuchsweise anders zu sehen.

Ganz ohne Wertung und Hierarchisierung: Literarische Texte, auch triviale, auch wenn sie bloß Unterhaltung oder (Ent-)Spannung versprechen, sind Teilexplikationen automatischer Deutungsmuster für Dinge und Verhältnisse, die wir kennen, die für uns »obvious« sind, die wir aber nicht erklären können. Sonst würde auch der trivialste Text nicht »funktionieren«.

Und noch mit einem weiteren, seit langem lieb gewonnenen linguistischen Vorurteil müssen wir aufräumen, so lehrt uns Burke in einem Text mit dem suggestiven Titel »What are the signs of what? (Burke 1966, S. 359-379). Mit großer Selbstverständlichkeit gehen wir davon aus, dass Sprachzeichen in irgendeiner (insgesamt doch recht dunklen) Weise Zeichen für die Dinge, Verhältnisse, Handlungen, Merkmale etc. sind, die wir »da draußen « lokalisieren und die sie für unsere Kommunikation und für unser Denken repräsentieren. Für die Analyse literarischer Texte ist ein solches Axiom (das erkennt man auf den ersten Blick) nur sehr bedingt hilfreich. Märchen, Sagen, Fabeln lassen sich einer solchen Axiomatik noch anpassen: Sie handeln eben von unserer Erfahrungsrealität im Modus der Tropisierung (Metapher, Metonymie, Synekdoche). Aber was machen wir mit der nicht minder prägnanten Erfahrung, dass ein und dieselbe Aussage, ein und derselbe »Satz« (siehe oben zu den Sprichwörtern und Redensarten!) zu höchst unterschiedlichen Situationen und Lebenslagen »passt «? Burke dreht den Spieß kurzerhand um und fragt: Was wäre, wenn wir probeweise einmal von der Gegenthese ausgingen, dass unsere Erfahrungen vielmehr die »Zeichen « sind, mittels derer wir das, was wir wahrnehmen, als Bestätigung, Herausforderung, Zusammenfassung unserer (per konzeptuellen von »terministic screens « angeleiteten und kumulierten) Deutungsordnungen verstehen und ratifizieren? Sein Beispiel ist, in der Literaturtheorie naheliegend, das Verhältnis einer Kapitelüberschrift, eines Novellentitels (das ließe sich leicht ausweiten auch für Zeitungstexte etc.) zu den Ausführungen im Textganzen:

If such verbal spirits, or essences, were enigmatically symbolized in nonverbal things, then their derivation (so far as causes within the natural world are concerned) could come both from the forms of language and from the group motives that language possesses by reason of its nature as a social product. This chapter examines both these possibilities. First, it proposes that language be viewed, not directly in terms of a word-thing relationship, but roundabout, 
by thinking of speech as an »entitling « of complex nonverbal situations (somewhat as the title of a novel does not really name one object, but sums up the vast complexity of elements that compose the novel, giving it its character, essence, or general drift). (Burke 1966, S. 361)

Die hochgradige Reflexivität unserer sprachlichen Praxis wird verdeckt durch den Umstand, dass wir sprechend stets glauben, in unmittelbarem Realkontakt mit dem »Besprochenen« zu sein. Die »Überschrift« (sei es die einer Erzählung oder die eines Zeitungstextes) lehrt uns, dass jede sprachliche Äußerung davon lebt, dass sie punktuelles Element in einer historisch-linearen Sequenz ist, die entweder früher oder später (meist: beides) in der Kette der Kommunikationen eingelöst werden muss. Und nicht wenige sprachliche Äußerungen, literarische wie alltägliche, sind Überschriften (»entitlements «) für vertraute Situationen der alltäglichen pragmatischen Orientierung. Das haben sie gemeinsam mit Burkes Sprichwörtern und Redensarten. Es versteht sich, dass es ein uraltes sprachphilosophisches Thema ist, das hier revitalisiert wird: die hoch reflexive »Abkürzungsfunktion« sprachlicher Zeichen gegenüber variablen Erfahrungen, die bereits in der Zeichenphilosophie des 18. Jahrhunderts (Leibniz, Wolff, Lambert) ein prominentes Thema war.

Der Sinn dieses kleinen Exkurses kann darum nur darin bestehen, zu erinnern, dass wir Literatur wohl gar nicht lesen könnten, wenn sprachliche Ausdrücke nicht auch als »Überschriften« (entitlement) komplexer Erfahrungssituationen gelesen werden könnten, die dann ihrerseits als Zeichen für unsere erlernten konzeptuellen Bezugssysteme fungieren. Burke selbst geht so weit, auch unsere gegenständliche Naturwahrnehmung für zeichenhafte Illustrationen sprachlicher Konzepte zu erklären, weil man Bedeutungen nun einmal nicht sehen kann:

But if the things of nature are, for man, the visible signs of their verbal entitlements, then nature gleams secretly with a most fantastic shimmer of words and social relationships. (Burke 1966, S. 379)

Fest steht jedenfalls, dass man in der Analyse literarischer Texte mit einer zünftig präzisen logischen Definitionssemantik, die uns sagt, wofür Sprachzeichen stehen, nicht weiterkommt. Was wir »unmittelbar « zu sehen glauben, ist eben nicht Teil der Lösung, sondern Teil des Problems, eben weil es das ist, was unsere terministic screens uns zu sehen erlauben.

\section{Textthesen}

a) Texte sind konnotative Systeme, und literarische Texte sind maximale entfaltete und elaborierte konnotative Systeme. Was ist darunter zu verstehen? »Konnotation « ist in der Linguistik ein schillernder und vieldeutiger Ausdruck. Der Sinn, in dem ich ihn hier gebrauche, lässt sich auf den dänischen Sprachtheoretiker Louis Hjelmslev zurückführen. Zeicheneinheiten (nicht etwa nur die Lexeme!) lagern neben ihrer Denotation (dem Bezeichneten bzw. dem Systemwert des Zeichens) in wechselndem Ausmaß die Spuren der kulturellen Praktiken an, in denen sie 
überwiegend verwendet werden (vgl. Maas 1985). Wenn ich von »Risiken und Nebenwirkungen« rede, dann ist konnotativ die Sphäre von Medizin und Medikamenten aufgerufen, weil die Formel da beheimatet ist. Und niemand kann mit $»$ Wahrlich, ich sage euch ...« anfangen, ohne das Neue Testament aufzurufen. Tue ich das in einer Vorlesung, so erziele ich den Effekt konkurrierender konnotativer »Rahmungen«: Vorlesung und religiöse Prophetie. Das, was häufig als Polyphonie literarisch-ästhetischer Texte apostrophiert wird, ist (so meine These) ein sem- und konnotationssynthetisches Arrangement von konnotativen Beständen, Perspektiven, Einstellungen.

b) Der typische literate Text adressiert in aller Regel einen »generalisierten Anderen « (George Herbert Mead; vgl. Maas 2010). »Literat « wohlgemerkt, nicht literarisch, und literat heißt nur »schriftkulturell«. Das heißt aber nicht, dass die fragliche Generalisierung grenzenlos oder dass der generalisierte Andere einfach mit der Sprachgemeinschaft identisch wäre. Die Generalisierung steht vielmehr für ein Kollektiv, das durch sprachlich gebundene und im kollektiven Erfahrungsraum verankerte Erfahrungsbestände gebildet und definiert wird. Und auch innerhalb dieser community ist die Generalisierung immer zugleich auch eine kontrafaktische soziale Idealisierung. Das wird sie erst recht an den Grenzen und Übergängen zwischen verschiedenen fachdiskursiven communities. Texte stiften Verknüpfungen zwischen individuellen Erfahrungsräumen. Das scheint mir ein gute, heuristisch fruchtbare axiomatische Ausgangsdefinition. Wer etwa politische Epochenbegriffe, Selbstdeutungen etc. verwendet, der tut das mit der praktischen Idealisierung einer (natürlich so niemals gegebenen) Austauschbarkeit der Standpunkte. Sie beinhaltet, dass seine Adressaten, stünden sie da, wo er steht, den Begriff ebenso gebrauchen und verstehen würden wie er selbst. Das müssen wir tun, um kommunizieren zu können, aber es ist eine praktische Idealisierung. ${ }^{1}$

Coseriu (1981) argumentiert im Blick auf literarische Texte weitergehend: Die nämlich seien nicht auf einen generalisierten Anderen zugeschnitten (wie es z. B. ein wissenschaftlicher Text ist, der auf eine fachliche community zugeschnitten ist), sondern generell ohne dominierende Alterität. Das Sprecher-Ziel, verstanden zu werden, stehe nicht im Vordergrund. Vermutlich sind das Nachwirkungen eines emphatischen Werkbegriffes. Wer beim Schreiben gar nicht an seine Leser denkt, muss sich das jedenfalls leisten können.

Mit wachsendem zeitlichem und kulturellem Abstand zwischen Produktion und Rezeption verschärft sich diese Diskrepanz, ohne dass die praktische Idealisierung aufgegeben würde. Jeder kennt den Fall, dass ein naiver Rezipient meint, einen Text vollkommen zu verstehen, den er lediglich seinen eigenen Erwartungen und Präferenzen anpasst (und bei dem er keinerlei Mühe darauf verwendet, die Produktionsbedingungen der Sinnvorlage zu rekonstruieren). Ohne jede Rückkopplung mit dem Produzenten (bzw. mit dem verfügbaren Wissen über diesen) kann jeder Rezipient mit einem Text machen, was er will. Und schon der nächste andere Rezipient wird womöglich einen ganz anderen Sinn aus der Vorlage extrahieren und seinen

\footnotetext{
1 Karl Mannheim hat dafür in den 1920er-Jahren die wissenssoziologische Formel vom »konjunktiven Denken« in Kommunikationsgemeinschaften geprägt.
} 
Vorgänger der Willkür bezichtigen. Die Mahnung der Historiker, es gebe gegen alle historiographischen Deutungen ein »Vetorecht der Quellen«, wirkt da etwas hilflos, da nur eine interpretierte Quelle Vetorechte beanspruchen kann. Bei literarischen Quellen gilt es umgekehrt als Qualitätsausweis, wenn sie sich als »polyvalent« erweisen und auch in neuen kulturhistorischen Zusammenhängen noch (oder wieder) Interpretationsressourcen abwerfen. Ich halte wohlgemerkt all diese Weiterungen keineswegs für Chimären. Sie sind berechtigt und müssen nur methodisch eingehegt werden. - Kurz: aufbewahrt wird nicht der Sinn, sondern eine immer strittige, aber verbindliche Vorlage für die indexikalische Sinnkonstruktion. Wer mit historischen Texten arbeitet, der hat trivialerweise immer die doppelte Aufgabe, die in den Begriffen und Konstruktionen konnotativ aufbewahrte Innenperspektive der Produktion zu rekonstruieren und sie in Beziehung zu setzen zum aktuellen Erfahrungs- und Erwartungshorizont der Rezeption. Das steht in jedem Hermeneutiklehrbuch.

Kenneth Burke hat dafür die Formel, die »Fakten « eines Buches seien die Wörter, Ausdrücke und Sätze, aus denen es besteht:

People usually think that the nonsymbolic realm is the clear one, while the symbolic realm is hazy. But if you agree that the words, or terms, in a book are its »facts «, then by the same token you see there is a sense in which we get our view of deeds as facts from our sense of words as facts, rather than vice versa. (Burke 1964, S. 147)

Alles Andere und Weitere hängt an den inferentiellen Ketten, mit denen wir die textuellen »Tatsachen« verbinden können. Es versteht sich natürlich auch, dass die fraglichen »Tatsachen« semiotischer Natur sind und von den Rezipienten in Bezüge eingestellt werden (müssen), über die sie verfügen. Aber die entscheidenden »Tatsachen«, über die wir verfügen, sind die, welche (ausgehend von den textuellen $»$ Fakten $\ll)$ durch unsere terministic screens inferentialisiert und ratifiziert werden können.

\section{Methodologie I: geordnete Rekurrenz}

Wie nähere ich mich einem ästhetisch-literarischen Text? Die linguistische Kritik an den literaturwissenschaftlichen Intuitionen moniert eigentlich immer den Mangel an ausdrucksseitigem Halt für inhaltsseitige Interpretationen. Ohne einen solchen Halt bleiben die Deutungen immer ein wenig subjektiv, willkürlich, kontingent wie immer man es nennen möchte. Um mich einem literarischen Text halbwegs methodisch zuwenden zu können, brauche ich, muss ich die dominante Ebene (oder die dominanten Ebenen) der formalen Organisation des Textes identifizieren. Die zu finden, ist leicht in einem kurzen Gedichtlein des folgenden Typs:

Per se bleibt beim Duell man gern per Sie

Denn die Beziehung ist nicht permanent

Wer schießt schon auf Personen, die man kennt?

Per Du wär' man nicht halb so schnell perdu. 
Von Prosodik, Rhythmik, Versmaß rede ich nicht, natürlich gibt es auch hier geordnete Rekurrenz, die analytisch zu fixieren wäre. In diesem Gedichtlein (es wird sicher nicht den Weg in die kanonisierte Geschichte der Lyrik finden!) ist aber die geordnete Rekurrenz der Silbe per zunächst am auffälligsten. Sie findet sich, bildlich gesprochen, an den prosodischen Eckblöcken des Gedichts (per se - per Sie und per $D u$ - perdu) jeweils kombiniert mit assonanten starktonigen Elementen (/ze:/ - /zi:/, /du:/ - /dy:/, allesamt offene Vollsilben, die auch phonologisch markante Rekurrenzen haben, auf die ich hier nicht eingehe). Jedenfalls ist per in den Eckzeilen immer zugleich schwachtonig und zentral. Und eigentlich auch in den Mittelzeilen. In den beiden reimenden Innenzeilen eröffnet per jeweils eine dreisilbige Wortform. Beide Wortformen haben aber stark kontrastierende prosodische Profile: <pér-manént vs. Per-só-nen>. Im ersten Fall sind die Ränder der Wortform starktonig und die Mittelsilbe schwach, im zweiten ist es die Mittelsilbe, die starktonig ist, während beide Randsilben schwachtonig sind. Kurz: Die Rekurrenzmuster für die Silbe per und für ihre jeweiligen syntagmatischen Partner sind als Organisationsprinzip des Gedichts leicht zu erkennen.

Semantisch einigermaßen zusammengehalten wird der Vierzeiler von einer schwachen Isotopiekette aus Merkmalen, die sich auf die Dimension der Nähe bzw. Distanz in sozialen Beziehungen anordnen lassen. Darüber ist nicht viel zu sagen. Das Ganze ist eben darum nur mäßig kunstvoll, weil der qua überzufällig geordneter Rekurrenz gestaltete Inhalt (für die Gegenwart der Rezipienten) einigermaßen fern und konnotativ nicht sehr reich (und mit überständigen feudalen Inhalten aufgeladen) ist. Immerhin gibt es noch ein höher organisiertes grammatisches Muster in dem Vierzeiler: die beiden ersten Zeilen sind indikativische Aussagen, die dritte Zeile ist eine rhetorische Frage und die vierte eine verkürzte irreale Konditionalkonstruktion.

Geordnete Rekurrenz und Kontrast sind die heuristischen Einstiegspunkte für eine halbwegs strukturalistisch angeleitete Analyse literarischer Texte. Sie liefern gewissermaßen das Rohmaterial solcher Untersuchungen. Das obige Gedichtlein ist darum an der ästhetischen Untergrenze, weil sich die aufdringliche und prominente Rekurrenz zunächst nur auf die artikulatorische Ebene der Silbe bezieht.

Ästhetische Gewinne lassen sich nach mehreren Seiten erzielen: Man kann die Bedingungen der puren ausdrucksseitigen Rekurrenz verschärfen und inhaltsseitig lediglich die Bedingung einhalten, dass sich das Produkt auch konnotativ und semsynthetisch noch interpretieren lässt oder wenigstens diesen Anschein erweckt. Ich gebe Ihnen ein Anagramgedicht von Unica Zürn (1988, S. 56) als Beispiel:

\section{Aus dem Leben eines Taugenichts}

\section{Es liegt Schnee. Bei Tau und Samen}

Leuchtet es im Sand. Sieben Augen

Saugen Seide, Nebel, Tinte, Schaum.

Es entlaubt sich eine muede Gans.

Weiter kann man vermutlich die Entautomatisierung der sprachlichen Sinnproduktion nicht treiben als in Anagramgedichten. Jede Zeile muss aus den Buchsta- 
ben-Bausteinen der Vorlage (und nur aus diesen) kombiniert werden. Das ähnelt der Zwölftontechnik der Komposition in der Tradition Schönbergs.

Die zweite Option besteht darin, die Struktureben der Rekurrenz in den Bereich der Zeichenphänomene zu erhöhen, mindestens in die Ebene der Morpheme (der kleinsten sprachsystemischen Zeichen aus Ausdruck und Inhalt), höchstens in die Ebene der Konstruktionen und der semantischen Merkmale (Isotopieketten). Im Bereich der Grammatik, also zwischen Morphemen und Konstruktionen, finden wir Phänomene, die (auf sehr unterschiedliche Weise) bedeutungshaltig sind, von denen ein einzelsprachlicher Zwang ausgeht. Der linguistische Strukturalismus rechnet alles das zur Grammatik einer Einzelsprache, was obligatorisch kodiert werden muss.

Grammatische Kategorien und Konstruktionen funktionieren im Gedicht bzw. in seinem Aufbau vor allem durch Verdopplung, Wiederholung und Kontrast (Jakobson/Pomorska 1982, S. 100). Von überzufälligen Laut-, Buchstaben-, Silbenkonfigurationen bewegen wir uns damit in das Feld der überprägnanten grammatischen Figuren.

Als Beispiel gebe ich hier einen Sechszeiler von Thomas Brasch (2013, S. 133)2, der ganz um die geordnete Rekurrenz grammatischer Figuren und Morpheme herum gebaut ist:

Was ich habe, will ich nicht verlieren, aber wo ich bin will ich nicht bleiben, aber die ich liebe, will ich nicht verlassen, aber wo ich lebe, da will ich nicht sterben, aber wo ich sterbe, da will ich nicht hin:

Bleiben will ich, wo ich nie gewesen bin.

Auf den ersten Blick sichtbar ist der Umstand, dass in jedem Vers die 1. Person Singular des Modalverbs »wollen« vorkommt. Fünfmal in negierter Form (will ich nicht) und einmal positiv (will ich) im letzten Vers. Alle Zeilen folgen dem gleichen grammatischen Konstruktionsmuster. Es gibt jeweils einen mit will modalisierten Matrixsatz, dessen Prädikat bzw. Hauptverb eine Ergänzung (teils grammatisches Objekt, teils Adverbiale) hat, die in der Form eines Relativsatzes realisiert ist. Fünf der sechs verbabhängigen Relativkonstruktionen sind mit einem Fragepronomen eingeleitet (vier mit lokalem wo, einer mit was). Nur der dritte Vers hebt mit einem »echten« Relativpronomen an. Die Linguisten werden dazu anmerken, dass Relativpronomina und Fragepronomina aus naheliegenden Gründen sehr enge Verwandte sind. Relativsätze »antworten « auf Fragen nach referentieller Spezifizierung. In den ersten fünf Versen bilden die abhängigen Relativsätze die erste Hälfte des Verses. Nur in der letzten, der im Matrixsatz nicht negierten Zeile tauschen Matrixsatz und Relativsatz die Positionen. Das ganze Gedicht lebt vom Kontrast zwischen den fünf Versen, die mit einer solchen offenen Relativkonstruktion beginnen, und dem sechsten Vers, der mit dem gewichtigen Hauptverb des Matrixsatzes (bleiben) anhebt und den (substantivierten und negierten) Relativsatz an den rechten Rand schiebt. In allen vorangehenden Versen steht die Negation im Haupt- und Matrixsatz, nur

\footnotetext{
${ }^{2}$ Es handelt sich um Abschnitt 6 aus »Der Papiertiger«.
} 
im Schlussvers wechselt sie in den Relativsatz, der zugleich nur dort die Position mit dem Matrixsatz wechselt. Vor dem Hintergrund der Bedeutung dieses letzten Hauptverbs ist die Dominanz lokaler Relativkonstruktionen wesentlich. In der hier aktiven Bedeutung fordert bleiben eine lokale Ergänzung. Bleiben kann man bekanntlich nur da, wo man ist oder war. So dass der letzte Vers, der mit bleiben will $i c h$ zunächst einen (sagen wir) versöhnlichen Schluss, eine Art Auflösung der vielen Negationen und treibenden aber-Sätze erwarten lässt, deren Ausweglosigkeit dann sogar noch verstärkt: durch einen Satz, der zwar einen Ort angibt, aber einen Ort, der mit dem von bleiben geforderten Argument semantisch unvereinbar ist. Und von dem wir zudem nicht wissen, ob es ihn (für das lyrische Ich) gibt oder ob er bloß das noetische Korrelat einer indirekten Frage ist.

Die ersten vier Verse enden auf adversatives aber, das zugleich den Relativsatz der Folgezeile vor-einleitet, wodurch ein stark treibender Effekt entsteht. In der Literaturwissenschaft spricht man von »Enjambement«, wenn ein grammatisch wesentlicher Teil der nächsten Zeile in die vorangehende genommen wird.

Nur antippen kann ich hier die grammatischen und sprachphilosophischen Tücken des Satztyps, von dem Brasch hier reichen Gebrauch macht. Sätze wie wo ich sterbe, wo ich lebe, wo ich bin sind nach ihrer Form zugleich indirekte Fragesätze und Relativsätze. Elemente wie wo, was, wie rechnen zugleich zu den Relativadverbien und zu den Frageadverbien. Je nach ihrer syntaktischen Umgebung fungieren solchermaßen eingeleitete Sätze als Relativsätze oder als indirekte Fragesätze oder als beides zugleich (Eisenberg 1999, S. 313 f.). Für den letzteren Fall (= zwei Lesarten) gibt Eisenberg den Beispielsatz:

\section{(1) Monika vergisst, was Manfred ausgesucht hat.}

Vergessen kann indirekte Fragesätze regieren. Dann wäre die Lesart ungefähr bedeutungsgleich mit Monika weiß nicht mehr, kann sich nicht erinnern, was Manfred ausgesucht hat. Lesen wir den Satz dagegen als Relativsatz zu einem gewöhnlichen Objekt von vergessen, dann müssen wir paraphrasieren mit: Monika vergisst das Objekt, das Manfred ausgesucht hat. Etwas zugespitzt: Solche Sätze können sich auf reale, identifizierte Objekte, Orte etc. beziehen (nach dem Muster attributiver Relativsätze) oder sie sind lediglich propositionale Konzepte, die nach solchen fragen. Sie können »gegenstandsfundierend « sein oder lediglich »propositionsfundiert«. Braschs Schlusszeile lässt genau diese Ambivalenz bestehen. Und sie ist es, die gewissermaßen die Pointe des Gedichts bildet. ${ }^{3}$

Es versteht sich, dass in diesen Zeilen Braschs noch viel mehr »verborgene Ordnung « steckt, als hier gezeigt werden kann. Ich nenne nur noch die Abfolge der Hauptverben: verlieren - bleiben - verlassen - sterben - sterben - bleiben.

\footnotetext{
3 In Wittgensteins Philosophischen Untersuchungen findet sich (wie Eisenberg 1999, S. 314 notiert) ein Passus, der auf den täuschenden noetischen Doppelcharakter solcher Relativ- wie Fragesätze hinweist. Was für die Sprache als Erkenntnisinstrument eine Quelle von Täuschungen und Missverständnissen sein kann, das ist für literarische Texte eine willkommene Ressource der Sinndiffusion und der »Polyvalenz«.
} 


\section{Methodologie II: Evokation und Konnotation}

Es wäre reizvoll, einigen Spuren in Braschs Sechszeiler genauer nachzugehen, ich möchte aber noch kurz auf zwei weitere linguistisch aufklärbare Dimensionen literarischer Texte hinweisen, die ich (Coseriu 1981 folgend) als »Evokation « und »Konnotation« bezeichne.

Als »Evokation « fasst Coseriu alle Effekte zusammen, die auf Beziehungen und Beziehbarkeiten zwischen den Zeichen des literarischen Textes und anderen Zeichen, Zeichenkategorien und Zeichensystemen beruhen (bis hin zu den Effekten, mittels deren ein Zeichen auf andere Texte verweist, oft als »Intertextualität « zusammengefasst). Als »Konnotation « fasst Coseriu alle Effekte zusammen, die auf Beziehungen zwischen den Zeichen und den kulturellen Praxisfeldern beruhen, in denen sie verwendet werden. Die Trennung beider Bereiche ist nur idealtypisch zu haben, meist vermischen sich beide Bereiche.

Ein prototypisches Verfahren von Evokations- und Konnotationssynthesen kann man illustrieren anhand eines (ziemlich literarischen) frühen Textes von Bob Dylan. ${ }^{4}$ In Bob Dylan's 115th dream legt der Text drei für die US-Geschichte mythische Schiffsfahrten aufeinander. Natürlich sind Schiffe schon selbst starke Kollektivsymbole, aber wenn ein und derselbe Text gleichzeitig die Mayflower (das Schiff der Pilgerväter von 1620, mit dem die Besiedelung und Kolonisierung der USA begann), die Pequod aus Herman Melvilles Roman Moby Dick mit ihrem besessenen Waljäger-Kapitän Ahab (im Lied heißt er »Arab«) und die Flotte des Christopher Columbus aufruft, dann lassen sich drei ziemlich differente Bezugssysteme aufeinander legen. Dylan macht Ahab zum Kapitän der Mayflower und lässt ihn das bigotte, aggressive und geschäftstüchtige Amerika der Gegenwart »entdecken«, wo sie gleich verhaftet und eingesperrt werden (weil sie Harpunen dabeihaben). Der ziemlich bekiffte (Alb-)Traum erzählt dann den Versuch des lyrischen Ich, für die Eingesperrten Hilfe bzw. Kaution zu organisieren. Dabei passieren dann Dinge wie das folgende: Das Ich betritt ein Gebäude, auf dessen Fassade mit dem Slogan $»$ Brüderlichkeit « geworben wird (»right across from a building advertising brotherhood «). Es stellt sich als Bestattungsunternehmen heraus, und der Inhaber quittiert die Bitte um Hilfe für die Freunde mit einer Visitenkarte und dem Satz: »Call me if they die $\ll$.

Nachdem dann das mit Ahab und der Mayflower gelandete Ich dem modernen US-Albtraum entfliehen kann, kommt in der Schlussstrophe Christopher Columbus zu Wort:

But the funniest thing was

When I was leaving the bay,

I saw three ships a-sailing

They were all heading my way

I asked the captain what his name was

And how come he didn't drive a truck.

\footnotetext{
4 Nach der (höchst unzuverlässigen!) Version in Dylan (1987, S. 468-475).
} 


\section{He said his name was Columbus}

I just said »Good luck.«

Die »Entdeckung « Amerikas rückt ans Ende und provoziert einen sarkastischen Kommentar. Die drei mythischen Schiffe, der Entdecker, die Pilgerväter und der Walfänger, bilden so etwas wie eine konnotative Rahmung, ein framing, eine Exposition (und zugleich eine Kontrastfolie) für die berichteten (traumentfremdeten und surrealen) Geschehnisse aus der Gegenwart des Balladen-Ich. Der literarische Effekt entsteht durch die Überlagerung der evokativen und konnotativen Sedimente aus dem Narrativ und aus den mythischen Rahmungen.

Ein zweites, anders gelagertes Beispiel: Volker Brauns Hinze-Kunze-Roman von 1985 macht in vielen Hinsichten kunstvollen Gebrauch von Techniken der sprachlichen Überlagerung unterschiedlicher (und gegensätzlicher) Konnotations- und Evokationssphären. Das Ergebnis, gerahmt durch die intertextuelle Dauerpräsenz von Diderots Jacques, le fataliste et son maitre und von Hegels Herr-Knecht-Dialektik (die zusammen den konnotativen Rahmen bilden), sind Textpassagen, die immer in mehreren über einander gelegten Ebenen zugleich bündig werden. Berichtet wird aus dem Alltag des SED-Funktionärs Kunze und seines Fahrers Hinze (bzw. umgekehrt: aus dem Alltag des Fahrers Hinze und »seines« Funktionärs Kunze). Dauerpräsent im Text ist aber auch der Erzähler selbst, der seine Aufgabe und deren Überwachung durch die Zensur und »Hauptverwaltung « permanent einarbeitet. Der erste Abschnitt des Romans exponiert die Formeln und Motive, deren indexikalische Streubreite dann im Folgenden entfaltet und ausgelotet wird:

Was hielt sie zusammen? Wie hielten sie es miteinander aus? Ich begreife es nicht, ich beschreibe es. Und immer der eine mit dem andren, und der andre machte mit? So verhielt es sich, was weiß ich; verflixt und zusammengenäht. Wenn man sie fragte, antwortete der eine für den anderen und der andere mit: Im gesellschaftlichen Interesse.

Aha, natürlich, erwidere ich: das Ding, um dessentwillen ich schreibe. (Braun 1985, S. 7)

$\mathrm{Zu}$ den exponierten organisierenden Textfaktoren gehört das Ensemble der Konstruktionen mit dem passe-par-tout-Verb halten: »Wie hielten sie es? Wie hielten sie es miteinander? Wie hielten sie es miteinander aus? (bis hin zu: Sie hielten es miteinander. Aus; S. 190). Ebenfalls im Anfang exponiert und immer wiederkehrend: die Opposition von begreifen (bzw. nicht begreifen!) und beschreiben.

Immer wieder ironisch und spöttisch eingeflochten in den Text wird (qua Erzähler-Ich) die (im gesellschaftlichen Interesse bestehende) Pflicht zum »Realismus« und zur Abstimmung mit der Zensur, die dadurch zugleich dargestellt, entlarvt und ridikülisiert wird:

Nach diesem sehr persönlichen Anfang, der mit der Hauptverwaltung abgesprochen ist, wenden wir uns der eigentlichen Handlung zu, der wesentlichen Darstellung der Wirklichkeit. Dies erfordert einen Realismus, der den großen Atem unserer Zeit hat. Zwar gibt es genug Gegenstände, vor denen uns der Atem stockt; man sieht es unsern hochroten, ewig röchelnden Romanciers an. 
Aber ich habe keine Wahl, ich muss mich an das Leben halten, das unsere Helden führen. (Braun 1985, S. 14)

Formeln wie »wesentliche Darstellung der Wirklichkeit« oder »großer Atem unserer Zeit« verweisen erkennbar auf den offiziellen slang der DDR-Literaturpolitik in den 1980er-Jahren.

Das Bauprinzip des Hinze-Kunze-Romans ließe sich ungefähr so umschreiben: Es gibt ein Netz wiederkehrender und wiederholter Propositionen, die durch ihre ständig neue Platzierung in den Umfeldern der Narration jeweils neu indexikalisiert werden und sich dadurch mit (überwiegend ironisch-satirischen) Sedimenten anreichern und aufladen. Zudem dürfte es für Sprach- und Literaturwissenschaftler gleichermaßen spannend sein, die (noetischen) Effekte zu registrieren und zu systematisieren, die aus minimalen Umstellungen, Veränderungen und Verschiebungen des nämlichen sprachlichen »Materials « resultieren.

Einen der vielen Höhepunkte dieser evokativen Engführungen bildet dann ein Passus, in welchem der Autor (das Erzähl-Ich) erzählt wie er, »im gesellschaftlichen Interesse «, sich der Diskussion stellen zu müssen glaubt (nach einem ziemlich anzüglichen Passus, in welchem wir erfahren, dass und wie der - ohnehin als pathologischer Schürzenjäger portraitierte - Funktionär Kunze seinem Fahrer Hinze die Frau ausgespannt hat - und wie der Fahrer darauf reagiert) - was natürlich der Literaturhauptverwaltung, so die geäußerte Vermutung, wenig konstruktiv und kaum vorbildhaft vorkommen möchte:

Als ich so weit gekommen war, hielt ich es, im gesellschaftlichen Interesse, für nötig, mich der Diskussion zu stellen, in einem Gremium, das ich im selben Interesse nicht näher beschreibe. Ich las ein Kapitel vor - der Einfachheit halber und um keine Zeit zu verlieren dieses, das ich gerade schreibe und das damit beginnt, dass Frau Prof. Messerle von einem anderen Buch sprach, das ich hätte schreiben sollen: obwohl es kürzlich ein anderer geschrieben hat. Sie konnte nicht genug gleichartige Bücher bekommen, musterhafte, sie stellte sie vermutlich im Wohnzimmer nebeneinander, eine sichere Bastion gegen die unzuverlässige Wirklichkeit. Aber wo mein Buch hätte stehen können, klaffte eine kleine Lücke; in der sie nun mit dem Zeigefinger fuhrwerkte; wodurch die sich aber, im Verlauf dieses Kapitels, noch erweiterte! Ich konnte nichts dafür, diese Zuarbeit wiederfährt den Realisten von unerwartetster Seite. (Braun 1985, S. $147 \mathrm{f}$.

Es dauert einen Moment, bis man die Travestie bemerkt, die darin besteht, dass der Bericht des Autors über die Anhörung bei der »Hauptverwaltung « zugleich der dort zur Diskussion gestellte Teil des Romans ist. So viel »Realismus« ist kaum $\mathrm{zu}$ überbieten. Was der »Hauptverwaltung « zur Diskussion vorgestellt wird, was sie adressiert, ist zugleich ein spöttischer Bericht über die »Hauptverwaltung «. Ich begreife es nicht, ich beschreibe es! Da wundert es einen nicht, dass es erhebliche Mühen gekostet hat, diesen Text in der DDR zum Druck zu bringen. (Die OstAuflage war, wie man nachlesen kann, winzig und augenblicklich ausverkauft, was aber bekanntlich das Interesse und die Zirkulationsgeschwindigkeit eines Textes unter DDR-Bedingungen eher noch steigert; gegen den damaligen stellvertretenden 
Kulturminister der DDR, der die Druckgenehmigung erteilt hatte, wurde ein Disziplinarverfahren eröffnet.) Im Schlusspassus des Romans wird die Travestie zwischen fiktiver Erzählung und realer Realität dann noch einmal gesteigert durch die Passage, die den Autor durch die Lottumstraße führt (hier spielt ein wesentlicher Teil des Romans). Da hat der literarische Verweis auf die heruntergekommenen Wohnverhältnisse die ganz reale Restaurierung der Altbauten hervorgerufen. Kurz: Wir wissen als Leser nie, ob wir uns gerade in der literarischen Fiktion befinden oder aber in der Realität, von der »realistisch « gerade berichtet wird.

Ich schließe den Abschnitt mit einem kleinen Gedicht (des großen Bertolt Brecht, zitiert nach Gernhardt 2010, S. 335), das einen wunderbaren Sphärenmix zwischen Alltag und Philosophie etabliert:

\section{Xantippe sprach zu Sokrates: \\ »Du bist schon wieder blau.« \\ Er sprach: »Bist du auch sicher dess'? \\ Kein Mensch weiß was genau." \\ Er gilt noch heut als Philosoph \\ Und sie als böse Frau.}

Die erste Zeile (»X sprach zu $\mathrm{Y} «$ ) ruft ein Spottgedicht-Format auf. Im mittleren Passus dieses Gedichts ist alltagssprachlich (und intertextuell) angespielt der Satz, für den auch in der (relativen) Breite Sokrates heute noch bekannt ist: »Ich weiß, dass ich nichts weiß«. Der ist alltagssprachlich reformuliert (und rhetorisch-pragmatisch gegen Xantippe gewandt) in der Formel: Kein Mensch weiß was genau. Der Alltag tritt in philosophischer Sprachform auf, die Philosophie in alltäglicher. Gleichzeitig führt der Text den alltagspraktischen Nutzen der skeptischen Philosophie vor (sie dient der Absicherung und Rechtfertigung eigenen Fehlverhaltens).

Was wir hier haben, ist Muster und Paradigma einer konnotativen und evokativen Überblendung zwischen zwei Sinn- und Praxissphären, die konventionell als maximal disjungiert gelten: der »niedere « Sphäre der alltagssprachlichen Verständigung und der »hohen« Sphäre der Philosophie.

\section{Entautomatisierung}

Zu den höchst auffälligen Merkmalen des Sprechens und des Redeverstehens gehört der weitestgehend automatische, mühe- und aufwandslose und aufmerksamkeitsentlastete Charakter beider Operationen. Und das bei einer formalen Komplexität der sprachlichen Darstellungstechnik, die auch nach Jahrtausenden fachlicher Bemühung keineswegs vollständig beschrieben oder gar erfasst ist. Während wir sprechen, sind wir mit unserer gesamten Aufmerksamkeit nicht bei der Sprache, wir haben sie vielmehr weitestgehend frei für das Gemeinte, für das, worüber gesprochen wird. Coseriu notiert das in anderen Worten auch im Eingangszitat. Der Sprache selbst wendet sich unsere Aufmerksamkeit immer nur dann zu, wenn der Weg zum Gemeinten blockiert ist, wenn uns der richtige Ausdruck fehlt, wenn wir im Unklaren über die Reichweite geteilten Wissens sind, also wenn Probleme mit dem Gemein- 
ten auftreten. Es »gehört « phänomenologisch zu sprachlichen Ausdrücken (in Texten und außerhalb von Texten!), dass sie intentional auf etwas zeigen oder verweisen, was sie nicht selbst sind. Die linguistische Semantik erkennt dieses Faktum an, verwendet dafür aber den (zumindest für Literaturanalysen!) schwerlich geeigneten Begriff der »Referenz«, der im Bezug (besser gesagt: in der Beziehbarkeit) sprachlicher Ausdrücke auf singuläre und identifizierbare Gegenstände der »Außenwelt« verankert wird - für die Alltagsrealität des Sprechens ein epistemologischer Grenzfall.

Literarische Texte (das ist durchaus eine minimalistische Charakterisierung) sind gegenüber dem alltäglichen Sprechen in hohem Maße entautomatisiert ${ }^{5}$ - allerdings zunächst nur, was ihre Produktion betrifft. Auch wenn es natürlich sowohl instinktiv schreibende als auch strategisch schreibende Autoren gibt, beide Typen »sprechen« in ihren literarischen Texten nicht so, wie sie im kommunikativen Alltag sprechen. Wenn sie auch im kommunikativen Alltag »literarisch « sprechen, dann wird das jeder als strategische Selbstinszenierung und als Selbststilisierung werten. Für die Rezeption literarischer Texte gelten selbstverständlich (sub-)kulturell höchst variable Maßstäbe. Unter den esoterischen Autoren der Moderne gibt es welche, die so hermetisch sind, dass man sie ohne Stift und Karteikarte kaum lesen kann, aber auch Autoren, die auf Rezipienten zunächst »süffig « wirken, aber nachträgliche Reflexionen und Implikationsketten in Gang setzen, die einen lange beschäftigen.

Bei der Produktion literarischer Texte geht die bewusste Aufmerksamkeit auf den gesamten semiotischen Prozess, also zusätzlich zur Sphäre des Gemeinten auf die Gesamtheit der Beziehungen und Beziehbarkeiten der involvierten Ausdrücke, Zeichen und Konstruktionen, auf deren Evokations- und Konnotationspotentiale. Etwas anders sieht es in der Sphäre der Rezeption literarischer Texte aus. Obwohl deren kundige Lektüre Lernen und Anleitung voraussetzt (wie jeder Germanistikstudent weiß), gilt ein literarischer Text mit einigem Recht als schlecht, wenn er ohne fachliche Anleitung gar nicht rezipiert werden kann. Ideal ist er dann, wenn er den Leser mühelos und automatisch in die Einzelheiten einer Sinnsphäre führt, die für alltägliche und außerliterarische Erfahrungen nicht oder kaum zur Verfügung steht. Die wesentlich reichere Palette der Beziehungen und Beziehbarkeiten verschafft dem Rezipienten eine Sinnerfahrung, die ihm erlaubt, seine Umgebung und Alltagswirklichkeit in irgendeiner Hinsicht »mit anderen Augen« zu sehen. Die technischen und kompositorischen Details, mit denen der Autor die fraglichen Sinneffekte erzielt, muss (und sollte!) der Rezipient dabei gar nicht identifizieren oder gar explizieren müssen. Das bleibt die Aufgabe des Literaturwissenschaftlers. In der Erfahrung des kundigen Rezipienten bleiben die Techniken eine Art impliziten Wissens (man erkennt einen guten Text, wenn man ihn liest, aber man weiß nicht unbedingt, warum genau er »ein guter Text« ist). Die Literaturwissenschaft rekonstruiert explizit und deklarativ die Fähigkeiten (neudeutsch Kompetenzen), die ein kundiger Leser haben sollte, wenn er den fraglichen Text mit Gewinn lesen will. Im Idealfall »versteht« der Leser den Text auf Anhieb - und merkt dann, dass er bei weitem noch nicht alles verstanden hat.

\footnotetext{
5 »Entautomatisierung « wurde als ästhetischer Begriff geprägt von Viktor Sklovskij - allerdings im Blick
} auf die Literaturrezeption. 
Dass »Entautomatisierung « ein rundum problematisches und widersprüchliches Konzept bleibt, möchte ich abschließend mit einem Beispiel verdeutlichen, das noch einmal von Kenneth Burke (1966, S. 25-44) stammt. Zu Edgar Allan Poe's Gedicht The Raven, zweifellos ein extrem kunstvolles und hoch strukturiertes Werk, gibt es einen von Poe selbst verfassten Kommentar, der die Entstehungsgeschichte des Gedichts erzählt. Dieser Text trägt den anspruchsvollen Titel »The Philosophy of Composition«. In ihm schildert Poe, wie er das Gedicht (angeblich) nach einem methodisch fest strukturierten Bauplan Schritt für Schritt komponiert hat. Man findet hier Überlegungen wie die, dass am Anfang die Frage gestanden habe, was das melancholischste poetische Thema überhaupt sein könnte, und die Antwort lautet, der Tod einer wunderschönen Frau, erlebt und geschildert durch ihren (einsam zurückgebliebenen) Geliebten. Burke schreibt:

He would try to show, he said, >that the work proceeded, step by step, to its completion with the precision and rigid consequences of a mathematical problem<. (Burke 1966, S. 25)

Burke lässt kaum Zweifel daran, dass er an eine solche Selbstmystifikation des schöpferischen poetischen Prozesses nicht glaubt. Gleichwohl verteidigt er den Autor, indem er festhält, der sei hier einfach in die Rolle des Literaturkritikers geschlüpft, und dessen Aufgabe sei es, die impliziten »Prinzipien« nach Möglichkeit zu explizieren, denen das Gedicht als strukturiertes Werk folgt, die aber darum keineswegs zum expliziten Plan des Autors gehört haben müssten - auch wenn sie möglicherweise dafür taugen, dass der Autor den eigenen Genius mit ihrer Hilfe mystifiziert.

»Entautomatisierung « der Produktion kann also nicht heißen, dass die gesamten Ordnungen eines literarischen Textes einem vorentworfenen Plan folgen müssten. Die »Prinzipien « stecken vielmehr implizit im sprachlichen Werk selbst (Burke betont gerne, dass »principles« logische oder temporale »firsts« sind, mittels derer wir uns die Dinge ableiten und zurechtlegen). Und so wenig der naiv-automatische Sprecher ein Bewusstsein oder einen Plan hat von den (grammatischen und pragmatischen) »Regeln«, denen er doch sprechend »folgt«, so wenig muss der literarische Autor ein explizites Bewusstsein von den Prinzipien der Komposition haben, die in seinem Werk entdeckt werden können. Diese Prinzipien (»firsts«) können aber nachträglich aus den Texten extrahiert werden, denen sie zu Grunde zu liegen scheinen. Und das dürfte im Großen und Ganzen der Modus sein, der für die Produktion (ausdrucksseitig fixierter) kultureller Objektivationen gilt. Sie sind, evolutionistisch gesprochen, nicht das Produkt von »intelligent design «, können aber im Nachhinein darauf hin analysiert werden, was sie wirksam und erfolgreich gemacht hat. Erst in einer solchen Schleife entsteht die Möglichkeit strategisch geplanter Textproduktion. Und an diesem Punkt ist die Analyse literarischer Werke mit den Werkzeugen der Linguistik im Vorteil, denn die Linguistik hatte schon immer damit zu tun, dass sie explizieren musste, welchen Regeln die Sprecher natürlicher Sprachen »automatisch« folgen, wiewohl sie kein explizites Wissen über sie haben.

Das von Burke selbst favorisierte Modell der »Entautomatisierung « des Sprechens (und zwar sowohl für die Literatur als auch für die Wissenschaft) steckt in seinem heuristischen Leitbegriff »perspective by incongruity«. Was in der sprach- 
lichen Sinnproduktion formelhaft und routiniert zusammengehört, das macht uns blind, eben weil es automatisch und vertraut funktioniert. Als Gegenmittel empfiehlt er die experimentelle Demontage und Neukombination der verbalen Atome (exemplifiziert u.a. am literarischen Verfahren von James Joyce):

We might say that planned incongruity should be deliberately cultivated for the purpose of experimentally wrenching apart all those molecular combinations of adjective and noun, substantive and verb, which still remain with us. It should subject language to the same »cracking process « that chemists now use in the refining of oil. If science would be truly atheistic or impious to the last degree, it should try systematically to eradicate every last linkage that remains with us merely as a result of piety or innate propriety, and not because of its rationally established justification. (Burke 1984 [1935], S. 119)

Die Mechanismen der sprachlichen Sinnproduktion können nur freigelegt werden, wenn man die Zeichen kombiniert, die im täglich routinierten Gebrauch gerade nicht zusammengehören. ${ }^{6}$

Der kundige Rezipient literarischer Texte entwickelt qua kumulativer Leseerfahrung skills. Und die sind durchaus wieder automatisch. Es sind freilich (nicht unähnlich darin den alltäglichen Routinen des Sprechens und Verstehens) sekundäre Routinen und Automatismen, entstanden aus der Mechanisierung und Kombination von Verstehenshandlungen, die ursprünglich durchaus Aufmerksamkeit gefordert haben. Noch einmal anders formuliert: Der Produzent literarischer Texte treibt einen ungeheuren kognitiven und kompositorischen Aufwand - mit dem Ziel, dass ein kundiger Leser umso weniger Aufwand benötigt, um die komplexen Sinneffekte erfahren und genießen zu können. Verschärft wird in jedem Falle die grundlegende Asymmetrie zwischen Textproduktion und Rezeption. Wenn ein Text niedergelegt ist und zirkuliert, dann kann im Grundsatz jeder Rezipient mit ihm machen, was er will.

\section{Schluss}

Was die linguistische Analyse literarischer Texte von der literaturwissenschaftlichen unterscheidet? Ich sage es mal etwas bösartig: Eigentlich nur der Umstand, dass die linguistische Analyse mit ausdrucksseitig belegbaren, identifizierbaren und verifizierbaren Regularitäten der Textoberfläche beginnt. Ob das nun Stränge semantischer Merkmale sind (Greimas' Isotopien) oder andere Formen der geordneten Rekurrenz. Von da an ist sie nicht weniger abhängig von den Tücken der Hermeneutik als die literaturwissenschaftliche Deutung auch. Die Differenz ist allein methodologisch, sie betrifft den Anspruch, Deutungen ausdrucksseitig zu verankern (und wirft natürlich die Frage auf, ob es für Evokation, Konnotation, Umfelder von Zeichen vernünftige

\footnotetext{
6 Burkes Terminologie ist (sicher auch als Illustration für das Prinzip der Inkongruenz) durchaus eigentümlich. Unter »piety« (Frömmigkeit) fasst er nicht primär religiöses, sondern grundsätzlich symbolsystemkonformes Handeln.
} 
ausdrucksseitige Evidenzen gibt). In den vergleichsweise einfachen Beispielen, mit denen wir hier zu tun hatten, dürfte das der Fall sein - aber natürlich nicht immer.

Funding Open Access funding enabled and organized by Projekt DEAL.

Open Access Dieser Artikel wird unter der Creative Commons Namensnennung 4.0 International Lizenz veröffentlicht, welche die Nutzung, Vervielfältigung, Bearbeitung, Verbreitung und Wiedergabe in jeglichem Medium und Format erlaubt, sofern Sie den/die ursprünglichen Autor(en) und die Quelle ordnungsgemäß nennen, einen Link zur Creative Commons Lizenz beifügen und angeben, ob Änderungen vorgenommen wurden.

Die in diesem Artikel enthaltenen Bilder und sonstiges Drittmaterial unterliegen ebenfalls der genannten Creative Commons Lizenz, sofern sich aus der Abbildungslegende nichts anderes ergibt. Sofern das betreffende Material nicht unter der genannten Creative Commons Lizenz steht und die betreffende Handlung nicht nach gesetzlichen Vorschriften erlaubt ist, ist für die oben aufgeführten Weiterverwendungen des Materials die Einwilligung des jeweiligen Rechteinhabers einzuholen.

Weitere Details zur Lizenz entnehmen Sie bitte der Lizenzinformation auf http://creativecommons.org/ licenses/by/4.0/deed.de.

\section{Literatur}

Brasch, Thomas (2013): »Die nennen das Schrei«. Gesammelte Gedichte. Berlin.

Braun, Volker (1985): Hinze-Kunze-Roman. Leipzig (und zeitgleich Frankfurt/M.).

Burke, Kenneth (1964): Terms for Order. Bloomington, Ind.

Burke, Kenneth (1966): Language as Symbolic Action. Berkeley, L.A.

Burke, Kenneth (1973): The Philosophy of Literary Form [1941]. Berkeley, L.A.

Burke, Kenneth (1984): Permanence and Change. An Anatomy of Purpose [1935]. Berkeley, L.A.

Coseriu, Eugenio (1981): Textlinguistik. Eine Einführung. Tübingen.

Dylan, Bob (1987): Songtexte 1962-1985. Frankfurt/M.

Eisenberg, Peter (1999): Grundriss der deutschen Grammatik, Band 2: der Satz. Stuttgart.

Gernhardt, Robert (2010): Was das Gedicht alles kann: Alles. Texte zur Poetik. Frankfurt/M.

Heider, Fritz (1958): The Psychology of Interpersonal Relations. New York [Nachdruck: Hillsdale, N.J. 1980].

Ichheiser, Gustav (1930): Kritik des Erfolges. Eine soziologische Untersuchung [Critique of success: A study in sociology]. In: Forschungen zur Völkerpsychologie und Soziologie, Vol. 9. Leipzig.

Jakobson, Roman (1960): »Linguistik und Poetik«. Wiederabgedruckt auf Deutsch u.a. in: Jakobson, Roman (1979): Poetik. Ausgewählte Aufsätze 1921-1971. Frankfurt/M., S. 83-121.

Jakobson, Roman/Pomorska, Krystina (1982): Poesie und Grammatik. Dialoge. Frankfurt/M.

Maas, Utz (1985): »Konnotation«. In: Franz Januschek (Hg.): Politische Sprachwissenschaft. Zur Analyse von Sprache als kultureller Praxis. Opladen, S. 71-96.

Maas, Utz (2010): Orat und Literat. Grundbegriffe der Analyse geschriebener und gesprochener Sprache. Graz.

Neckel, Sighard (2008): Flucht nach vorn. Die Erfolgskultur der Marktgesellschaft. Frankfurt/M.

Zürn, Unica (1988): Gesamtausgabe, Band 1: Anagramme. Berlin. 